\title{
FRACTURE OF THE HEAD OF THE RADIUS.
}

\author{
By E. H. BENNETT, M.D., M.Ch., F.R.C.S.;
}

Professor of Surgery in the University of Dublin, \&c.

[Read in the Section of Pathology, May 30, 1890].

Tris example of fracture of the head of the radius which I desire to submit to the Academy of Medicine is of interest chiefly for its rarity. I regret that $I$ can give no life history of it, as I have obtained it from the body of an elderly woman during the course of operative surgery in the School of Physic this session.

A careful examination of the limb which contained it showed that no other fracture, either of the bones of the forearm or the humerus, was associated with it, nor was there any evidence that dislocation of the bones of the forearm had occurred at any time, for the structure of the several ligaments of the joint showed no trace of such accident.

The line of the fracture as it traverses the cartilage of the head of the bone is clearly marked. It divides the cup-shaped depression which articulates with the capitellum of the humerus into two almost equal parts, and passes down on either margin through the surfaces which articulate with the lesser sigmoid cavity of the ulna and the coronary ligament respectively, and can be indistinctly traced on the corresponding surfaces of the neck of the bone. There is no projection caused by callus on any part of the line, either in cartilage or bone, but the fracture has been firmly united by bone.

The line of fracture in the cartilage of the radial cup passes almost diametrically across it in a line parallel to the anterior surface of the shaft. The anterior half was evidently detached from the rest of the bone and displaced slightly beneath its fellow.

At first sight the position of the line of fracture, and the direction of the displacement of the anterior fragment, would suggest that the injury was the result of undue pressure breaking off the 
fragment, as the radius tended to be displaced directly backwards, as in cases of dislocation of both bones backwards at the elbow, under conditions in which the fracture has been found in association with fracture of the coronoid process of the ulna. The circumstances I have mentioned bear against this view.

If the bones of the forearm are fully pronated, and so viewed in relation to the articular surfaces of the humerus placed in relation with them, it is seen that when the joint is almost fully extended while the radius is pronated, the line of fracture marks off the surface of the unbroken cup, which is free of the capitellum posteriorly, from the half detached by facture, which, in this position, is in contact with the capitellum. It is very unlikely that a shock acting in the line of the axis of the radius would occur while the hand was held supine, while the prone position of the wrist with an extended elbow is more likely to be that in which such shock would occur.

This specimen is exceptional, like two or three of those already recorded, in being uncomplicated by fractures of the coronoid process of the ulna, complete or incomplete, or by fractures of the radius elsewhere, or of the humerus. In the absence of any life history it is useless to speculate further on the cause, except, perhaps, to note that there is here an entire want of evidence of any great injury such as has been commonly noted in the few recorded examples of the fracture; there is no fracture of any other bone of the body, nor anything to suggest that any great injury had caused the fracture.

Although completely united, and devoid of any traces of recent injury, the specimen shows one other point worthy of note. On the surface of the capitellum a trace of the degeneration of chronic rheumatic arthritis is present, not in its idiopathic form, such as one might expect to meet in an old woman, the inhabitant of one our workhouses, but a recently-established disease which has not yet worn through the cartilage of the capitellum, and which, not being present in the opposite joint, points to its being the result of the injury. Adopting this view, the accident must have occurred not more than a year or so before death. 